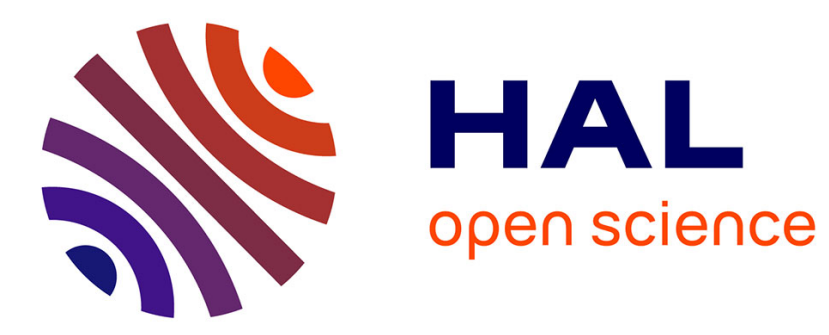

\title{
DIFFRACTION STUDIES OF THE ATOMIC STRUCTURE OF GRAIN BOUNDARIES
}

\author{
J. Budai, S. Sass
}

\section{To cite this version:}

J. Budai, S. Sass. DIFFRACTION STUDIES OF THE ATOMIC STRUCTURE OF GRAIN BOUNDARIES. Journal de Physique Colloques, 1982, 43 (C6), pp.C6-103-C6-113. 10.1051/jphyscol:1982611 . jpa-00222291

\section{HAL Id: jpa-00222291 https://hal.science/jpa-00222291}

Submitted on 1 Jan 1982

HAL is a multi-disciplinary open access archive for the deposit and dissemination of scientific research documents, whether they are published or not. The documents may come from teaching and research institutions in France or abroad, or from public or private research centers.
L'archive ouverte pluridisciplinaire HAL, est destinée au dépôt et à la diffusion de documents scientifiques de niveau recherche, publiés ou non, émanant des établissements d'enseignement et de recherche français ou étrangers, des laboratoires publics ou privés. 


\title{
DIFFRACTION STUDIES OF THE ATOMIC STRUCTURE OF GRAIN BOUNDARIES
}

\author{
J. Budai and S.L. Sass \\ Department of Materials Science and Engineering, Cornelz University, \\ Ithaca, NY 14853, U.S.A.
}

\begin{abstract}
Résumé. - Une revue des récents résultats obtenus sur la structure atomique des joints de grain par des techniques de diffraction est présentée. Pour 1a première fois, la structure d'un joint de grain est déterminée seulement à partir des données de diffraction. L' influence d'un métal particulier sur la structure de joint est aussi considérée. Par ailleurs, la variation de 1'épaisseur de joint de grain est établie en fonction de paramètres tels que 1'angle de désorientation et l'orientation du plan de joint. Grâce aux résultats d'études de diffraction, des généralisations sont avancées sur la structure des joints de forte désorientation.
\end{abstract}

Abstract. - The recent results of the application of diffraction techniques to study the atomic structure of grain boundaries are reviewed. The outcome of the first determination of a grain boundary structure using diffraction information alone is examined. The influence of a particular metal on the grain boundary structure is examined. The variation of grain boundary structural width with parameters such as misorientation angle and boundary plane is studied. Generalizations are made concerning the structure of large angle boundaries based on the results of the diffraction studies.

\section{Introduction}

There have been a large number of studies made using sophisticated experimental techniques to examine the structure of grain boundaries. Transmission electron microscopy studies have revealed the dislocation content of various boundaries (see, for example, Ref. 1) and the rigid body translation between the two crystals neighboring the boundary (2). For tilt boundaries, high resolution microscopy techniques have provided information with resolution approaching atomic dimensions $(3,4)$. Electron and $X$-ray diffraction studies have demonstrated through observations in reciprocal space that the boundary is periodic (5), that wel1 defined patterns of relaxation exist (6) and that the boundary structural width can be measured (7). To date, however, none of these experimental techniques have proved capable of determining, without ambiguity, the exact location of all atoms in the grain boundary region. On the theoretical side, sophisticated computer modelling techniques have been developed which can determine the detajled atomic structure of boundaries but which also suffer from a number of limitations. Most notable of these is the choice of a realistic interatomic potential.

As a step towards obtaining a believeable atomic structure, several studies have attempted to correlate experimental observations with structures generated by computer modelling. This approach has met with a certain degree of success with determinations of translation states (8), projected tilt boundary structures (9) and, most recently, of the complete core structure of a twist boundary (10). In the latter study the intensities of extra grain reflections from a $\Sigma=13^{*}\left(\theta=22.62^{\circ}\right)$ [001] twist boundary in gold measured with $X$-ray diffraction were compared with the structure factors of computer generated boundary structures. The present paper describes how the diffraction approach can be used to determine a grain boundary structure, presents the first determination of a boundary structure made using only diffraction information (11), compares boundary structures in different metals (12), discusses grain boundary structural width measurements, and then makes generalizations concerning the structure of large angle [001] twist boundaries based on diffraction observations.

\footnotetext{
$\Sigma$ is the reciprocal of the density of coincidence sites.
} 
In order to better understand the details of the experimental results, it is worthwhile to examine the reciprocal lattice associated with the grain boundary. A11 of the X-ray observations are made on manufactured bicrystais (see reference 13 for details), one of which is shown schematically with a small angle twist boundary at its midplane, in Fig. 1(a). The reciprocal lattice of this boundary is shown schematically in Fig. $\mathrm{I}(\mathrm{b})$, where the $\mathrm{H}$ and $\mathrm{K}$ axes 1 ie parallel to the boundary, and the L-axis is normal to the boundary plane. The periodic strain field associated with the grain boundary gives rise to extra reflections, which are shown in the form of reciprocal lattice rods (relrods) since the boundary is not periodic along the $z$-direction. Reflection intensities in the $L=0$ plane are related to the structure of the boundary projected onto the boundary plane while the length of the relrods is related to the boundary width. The structural information discussed in this paper comes from measurements made on either the structure factors in the $L=0$ plane $\left(F_{H K O}\right)$ or the relrod profiles along the $L$ direction.

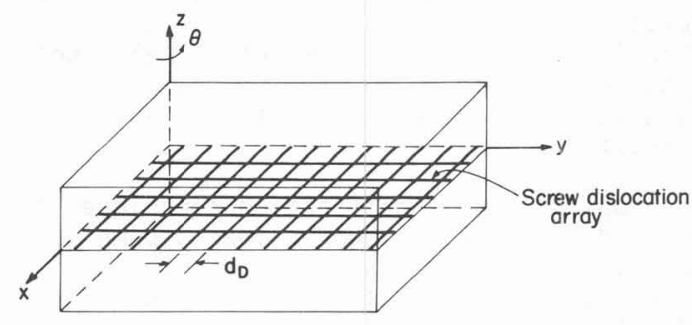

[a]

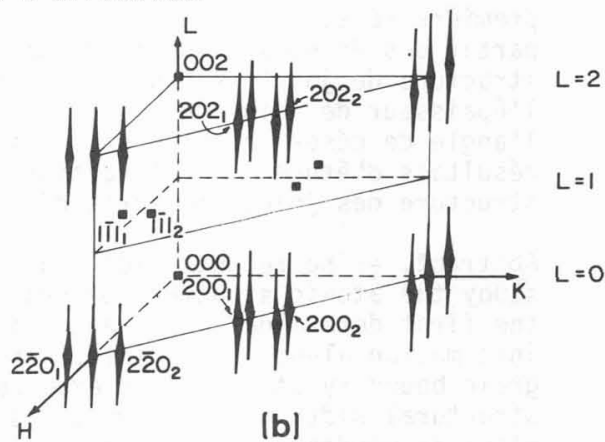

Figure 1. (a) Bicrystal containing a small angle [001] twist boundary with misorientation $\theta$ at its midplane.

(b) Schematic three-dimensional reciprocal lattice for the grain boundary in (a).

II. The Projected Structure of the $\Sigma=5\left(\theta=36.87^{\circ}\right)$ [001] Twist Boundary

A. The Symmetry of the Unit Cell

When determining the structure of a periodic object the first step is to determine the dimensions of the unit cell. In grain boundaries studies performed on manufactured bicrystal specimens, the unit cell is expected to be fixed by the choice of the misorientation axis and angle, and the boundary plane. The second step is to determine the symmetry elements of the unit cell.

Fig. 2(a,b) shows top and side views, respectively, of the unit cell of the $\Sigma=5$ twist boundary, with 2 atomic planes above and below the boundary plane. The structure shown is in the coincidence site lattice (CSL) configuration; that is, the two crystals are not translated away from the coincidence position for f.c.c. structures. Following Bristowe and Crocker (14), it is seen that this unit cell has the symmetry elements shown in Fig. 2(c). Any translation of one crystal with respect to the other, which is not a vector of the DSC lattice (15), wi17 change the symmetry associated with the boundary structure. Fig. 2(d,e) shows the symmetry elements of two high symmetry translated states characterized by DSC translations $\vec{D} 1 / 2$ and $\vec{B} 1+\vec{D} 2 / 2$ (where $\vec{D} 1$ and $\vec{D} 2$ are the orthogonal DSC basis vectors in the plane of the boundary (14)). Other translations are possible, and for these cases, the number of symmetry elements decreases. The symmetry of the unit cell can be determined from the experimentally observed structure factor rules. The structure factor rules for the three translation states shown in Fig, $2(\mathrm{c}, \mathrm{d}, \mathrm{e})$ are given in the Table. A complication in this analysis arises for the $\mathbf{D} 1 / 2$ translation state, where the actual boundary structure could consist of a mixture of domains with the same translation state, but different orientations. For example, in different areas of the boundary the translation can be either $\mathbb{D}_{1 / 2}$ or D2/2, yielding two domains. In this case the scattering from the two domains will average together in such a way that there are no longer structure factor forbidden reflections and more symmetry is shown in the diffraction pattern than actually exists in any particular domain. 


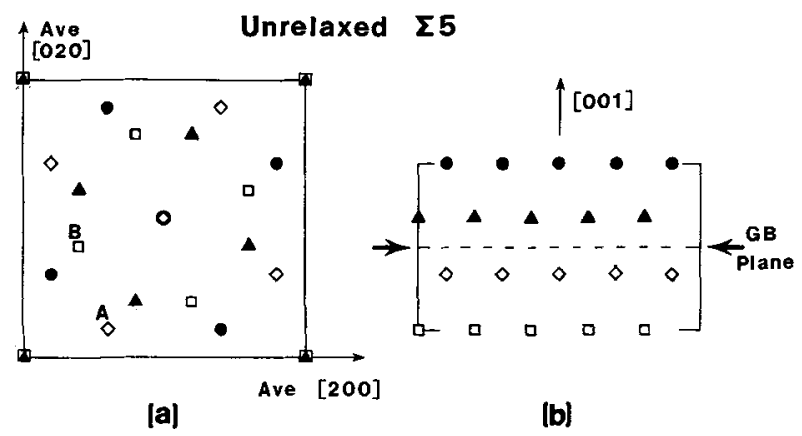

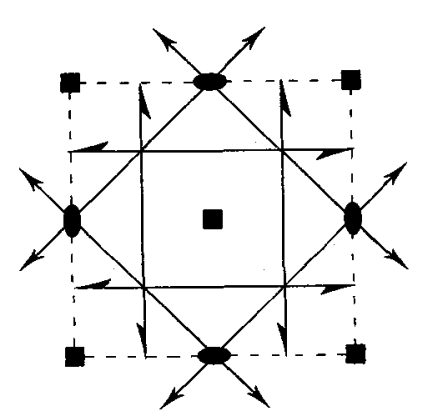

$|c|$

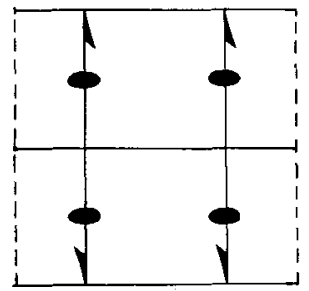

[d]

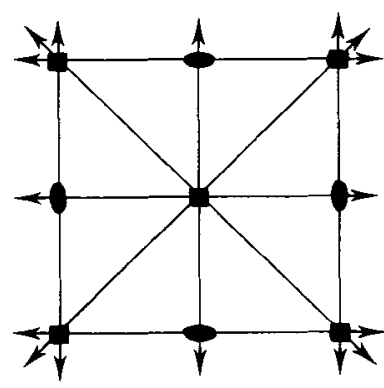

[e]

Figure 2. (a) Schematic diagram of the unrelaxed $\Sigma=5$ primitive CSL unit cell projected onto the grain boundary plane. (b) Side view of the same unit cell with two planes above and below the boundary shown. The symmetry elements of (c) CSL, (d) $\overrightarrow{D T} / 2$ translation and (e) $(\vec{D} 1+\vec{D} 2) / 2$ translation [001] twist boundaries in $\mathrm{fCC}$ crystals. The squares and ellipses represent four-fold and two-fold axes perpendicular to the interface; the continuous lines represent two-fold and two-fold screw axes in the interface, the latter being distinguished by half arrowheads. The diagrams are valid for all values of $\Sigma$.

Table: Diffraction symmetry and structure factor selection rules

CSL Structure

$\left|F_{H K L}\right|=\left|F_{H K L}-\right|=\left|F_{\overline{H K L}}\right|=\left|F_{H K L}\right|=\left|F_{H K L}\right|=\left|F_{K H L}\right|$

$F_{H O O}=0$ if $H$ is odd

$\mathrm{F}_{0 K 0}=0$ if $K$ is odd

DI $1 / 2$ Structure

$\left|F_{H K L}\right|=\left|F_{\overline{H K L}}\right|=\left|F_{\overline{H K L}}\right|=\left|F_{H \overline{K L}}\right|=\left|F_{H K L^{-}}\right| \neq\left|F_{K H L}\right|$

$F_{O K O}=0$ if $K$ is odd

$\left(\vec{D}_{1}+\vec{D}_{2}\right) / 2$ Structure

$\left|F_{H K L}\right|=\left|F_{\overline{H K} \bar{L}}\right|=\left|F_{\overline{H K L}}\right|=\left|F_{H \overline{K L}}\right|=\left|F_{H K L}\right|=\left|F_{K H L}\right|$

No structure factor forbidden reflections. 
The distinguishing difference between the CSL and all other configurations lies in those structure factor selection rules which are related to the presence of two-fold screw axes. For the CSL structure, $\mathrm{F}_{\mathrm{HOO}}(\mathrm{H}=\mathrm{Odd})$ and $\mathrm{F}_{\mathrm{OKO}}$ (K=odd) must be zero, whereas for the $(\bar{D} 1+\bar{D} 2) / 2$ translation or $\mathrm{HOO}_{\text {for a mixture }}$ okO domains with $\vec{D} 1 / 2$ and $\vec{D} 2 / 2$ translations, intensity can be present at these positions. The observations of Budai, Bristowe and Sass (11) are consistent with the $\Sigma=5$ unit cell being in the CSL configuration (untranslated state). The observations of Gaudig and Sass (13) (which were repeated in more detail in ref. 11) are consistent with the $\Sigma=13\left(\theta=22.62^{\circ}\right)$ [001] twist boundary also being in the untranslated state.

\section{B. The Number of Independent Atoms in the Unit Cell}

Once the symmetry has been established the next step is to determine the minimum number of atoms whose positions must be located in order to completely characterize the unit cell contents. By examination of Fig. 2(a) it is seen that there are 5 atoms per $\mathrm{plane}$, with the $x, y$ positions of one atom per plane (at either $1 / 2,1 / 2$ or 0,0 ) fixed by the translation state of the boundary. The remaining four atoms in the plane are symmetry related by the 4-fold axis. Thus it is necessary to locate 2 atoms per plane in order to locate all the atoms in the plane. If only the $x, y$ positions are to be determined, it is necessary to locate 1 atom per plane in order to locate all the atoms in the plane. It is also seen that the atoms in the upper and lower crystals are related by the two-fold screw axes lying in the interface plane. What this means is, if it is assumed that the grain boundary is 4 atomic layers thick, then in order to determine the atomic structure projected onto the boundary plane, it is necessary to determine the $x, y$ coordinates of only 2 atoms (marked A,B in Fig. 2(a)). Most of the experimental observations are in the $L=0$ plane (see Fig. $1(a)$ ), which contains information only on the $x, y$ coordinates. Thus the study here will be concerned with determining the projected boundary structure.

The assumption that significant atomic relaxations for a $\Sigma=5$ boundary are limited to a region which is only 4 atomic layers thick ( $0.8 \mathrm{~nm}$ for $A u$ ) is based on both experimental and theoretical considerations. The width of the strained region for a $\Sigma=377\left(\theta=23.8^{\circ}\right)$ twist boundary in gold was experimentally determined, from the intensity profiles of various relrods, to be $20.8 \mathrm{~nm}(16)$. The intensity profiles for relrods for a $\Sigma=5\left(\theta=36.9^{\circ}\right)$ boundary were qualitatively observed to be broader, implying a narrower width for the strained region. For the $\theta=23.8^{\circ}$ case, the boundary thickness was in good agreement with the 0-lattice spacing, do, of $0.7 \mathrm{~nm}$. This agreement is consistent with St. Venant's principle (17). For the $\Sigma=5$ boundary, the $0-7$ attice spacing is only $0.456 \mathrm{~nm}$ and a narrower boundary region is expected. If the stresses decrease exponentially as exp $\left(-2 \pi z / d_{0}\right)(17)$, then the displacements at a distance, $z$, corresponding to the third atomic $D_{p l a n e}$ from the boundary, would be a factor of 275 times smaller than the displacements in the first plane. Thus the assumption that significant relaxations are restricted to a four-layer region in the vicinity of the boundary plane is quite reasonable.

\section{The Atomic Positions in the Unit Cell.}

Following upon the conclusions of the previous sections, the determination of the projected $\Sigma=5$ boundary structure proceeds by the standard reliability factor approach that has been used for crystal structure determinations (18). In this method the $x$ and $y$ coordinates for the two independent atoms labelled $A$ and $B$ in Fig. 2(a) are scanned in small increments over an area covering the range of atomic displacements which generate all possible grain boundary configurations. For each configuration the magnitude of the structure factors are calculated and compared to experimentally observed structure factors using the definition of the reliability factor, R, given below,

$$
R=\sqrt{\frac{\sum W_{j}\left(\left|F_{j}^{o b}\right|-\left|F_{j}^{c a}\right|\right)^{2}}{\sum W_{j}\left(F_{j}^{o b}\right)^{2}}}
$$

where $F_{j}^{O b}=$ the observed structure factor of the $j$ th reflection. 


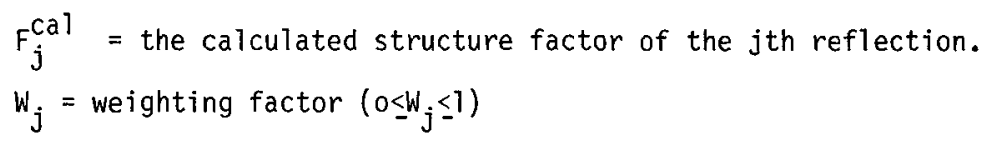

The set of $x_{A}, y_{A}, x_{B}, y_{B}$ coordinates which leads to the smallest value for $R$, provides the best fit to the diffraction observations. Thus the determination of the $\Sigma=5$ structure becomes a search for minimum values for the function $R\left(x_{A}\right.$, $\left.y_{A}, x_{B}, y_{B}\right)$. Such a search yields the $\Sigma=5$ structure shown in Fig. 3 .

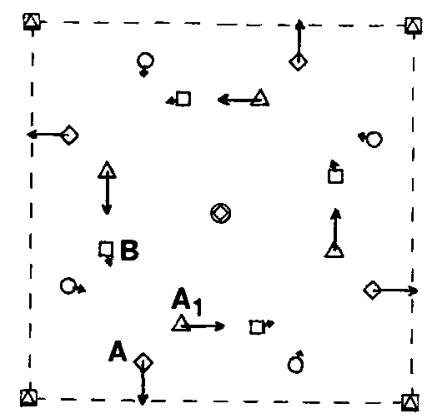

Figure 3.

Atomic displacements for the projected $\Sigma=5$ CSL unit cell. This structure had the smallest $R(=0.15)$.

Examination of Fig. 3 reveals several interesting points about the structure. Note that the displacement associated with atom $A$ (first plane from the boundary) is much larger than the displacement associated with atom B (second plane from boundary). This rapid decrease in magnitude for the atomic relaxations in planes away from the boundary is as expected for a large angle boundary. In order to understand the origin of the relaxations, consider the forces acting on atom $A$. The atoms in the lower crystal act to hold atom $A$ in its unrelaxed position in order to preserve f.c.c. stacking. The atoms in the upper crystal act to displace atom $A$, with the nearest atoms having the largest effect. In the unrelaxed configuration, atoms $A$ and $A_{1}$ are $23 \%$ closer than the nearest neighbor distance in a perfect f.c.c. crysta1. Thus the observation that in this structure these atoms move apart is physically quite reasonable. Examination of the boundary structure shows that it exhibits symmetry related displacements which can be interpreted as local rotations about ' 0 ' elements. For [001] twist boundaries, the ' 0 ' elements consist of rods lying perpendicular to the boundary, some of which pass through CSL sites $(15)$. The degree of rotation is large $\left(\cong 20^{\circ}\right)$.

At this point, it is important to attempt to understand the $\Sigma=5$ boundary structure in terms of more general physical concepts which may be applicable to other boundaries. The existence of rotational type relaxations in grain boundaries was first recognized in connection with low angle twist boundaries (1). Here, the observed structure can be interpreted as local rotations about ' 0 ' points of approximately $\theta / 2$ which produced 1 arge areas of near perfect lattice separated by narrow regions of misfit (lattice screw dislocations). Clearly, if local rotations of about $\theta / 2$ are to occur in large angle boundaries then large atomic displacements would be involved. Such displacements were not found to occur in the $\Sigma=5$ computer simulations (11) but were a result of the diffraction analysis, as can be seen in Fig. 3 .

Further insight into the unique nature of the relaxations associated with the structure generated by the diffraction analysis can be gained by examining Fig. $4(a)$ which shows six unrelaxed unit cells containing only the atoms in the first planes above and below the boundary. As mentioned earlier, the relaxation of any "white" atom depends on a balance between the repulsive forces of the nearby "black" atoms and the restoring force of the "white" single crystal. Figure 4(b) shows the structure determined by the diffraction analysis. The "white" atoms are located approximately on the edges of the CSL unit cell, positions of special symmetry. It can be seen that the magnitudes of the relaxations are such as to maximize the distance between the "white" and "black" atoms. 


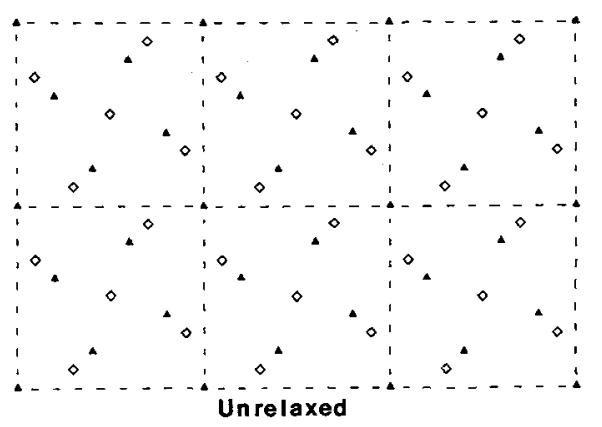

[a]

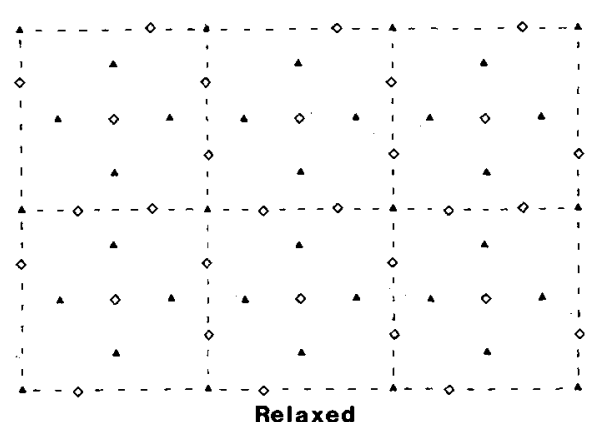

|b]

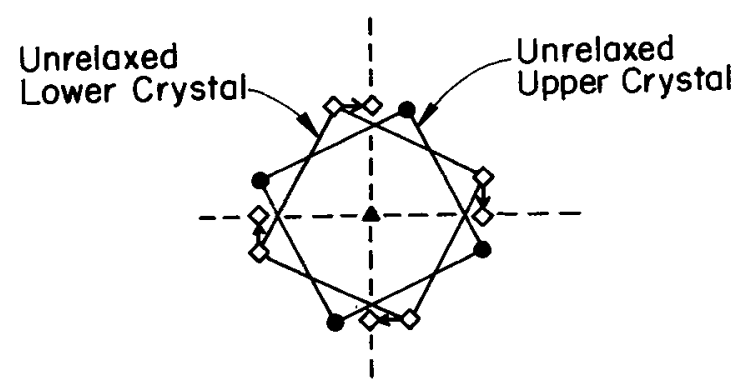

[c]

Figure 4. (a) Projected unrelaxed $\Sigma=5$ CSL unit cell containing only the atoms in the first planes above and below the grain boundary. (b) Projected relaxed $\Sigma=5$ CSL unit cell determined by the diffraction analysis. (c)

Illustrating the atomic configuration in the vicinity of a unit cell corner. 
In Fig. $4(c)$ the situation in the vicinity of a unit cell corner in Fig. $4(a, b)$ is shown but now with the 2 nd, 4 th, 6 th, etc., planes above the boundary indicated by a black circle $\bullet$ and the ist, 3rd, 5 th, etc., planes below the boundary indicated by a white diamond $\diamond$. Here it is seen that the $\diamond$ atom in the lst plane below the boundary has rotated by $\sim \theta / 2$ (indicated by the arrow) in a clockwise sense to a position which is approximately on the unit cell edge. It is clear that the displaced $\diamond$ atom is in the position of the median fcc unit cell between the upper and lower crystals. Thus the diffraction structure conforms to the concept, originally recognized in low angle boundaries, that defines the interface as discontiguous patches of median fcc structure. It is interesting that examination of the structure of the $\Sigma=13$ boundary determined by Bristowe and Sass (10) [Fig. 4(c) of their paper] shows the presence of the same local structure, since atoms above and below the boundary $p l a n e$ rotate about ' 0 ' elements by an angle of about $\theta / 2$. As a logical extension of these results, it is tempting to generalize the presence of patches of median fcc structure to ail high angle [001] twist boundaries.

III. Comparison of the Structure of Twist Grain Boundaries in Au and Ag (12) In a recent computer modelling study (10) the atomic structure of the $\theta=22.62^{\circ} \quad(\Sigma=13)[001]$ twist boundary was calculated for several f.c.c. metals. It was predicted that the structure of this type of boundary should be different in different metals. Since the properties of a boundary are expected to be dependent in some manner on the details of its structure, it is particularly important to check the validity of this prediction. This was done by comparing the structure of the $\Sigma=13$ [001] twist boundary in Au and Ag using X-ray diffraction techniques. Specifically, X-ray diffraction patterns showing the same regions of reciprocal space were obtained and compared for $\mathrm{Au}$ and $\mathrm{Ag}$ bicrystal specimens which contain the $\Sigma=13$ twist boundary. The intensities of the reflections in these patterns are related to the square of the structure factors of these reflections. It is expected that if the boundary structures are significantly different, then the relative intensities of the grain boundary reflections observed from the $\mathrm{Au}$ and $\mathrm{Ag}$ boundaries will also be different.

Since the lattice parameters of $\mathrm{Au}$ and $\mathrm{Ag}$ are approximately the same (ao $=$ $0.40783 \mathrm{~nm}$ for $\mathrm{Au}$ and $\mathrm{ao}=0.40856 \mathrm{~nm}$ for $\mathrm{Ag}$ ), the diffraction geometry is the same for both metals. It is possible then to search for any differences in the structure factor by simply examining the same type diffraction patterns from the $\mathrm{Ag}$ and $A u$ specimens and looking for differences in the relative intensities of particular grain boundary reflections.

Examples of the experimental observations are presented in the form of pairs of X-ray diffraction patterns taken in the vicinity of the f.c.c. reflections in Fig. 1(b) with the same diffraction geometry for the Au and Ag grain boundaries. Fig. 5(a,b) shows patterns for $\mathrm{Au}$ and $\mathrm{Ag}$, respectively, taken in the vicinity of the $200 \mathrm{f} . \mathrm{c} . \mathrm{c}$. reflections, with grain boundary reflection $6,6,0$ on the Ewald sphere. The indices of the grain boundary reflections are indicated on the patterns in Fig. $5(a, b)$ using the $H, K$ notation for the CSL unit cell. Clearly present in the Au pattern are several grain boundary reflections. These can be compared to the pattern from $\mathrm{Ag}$ in Fig. 5(b) and it is seen that the relative intensities of the reflections in the two patterns are quite similar. For example, listing the observed reflections in decreasing order of intensity yields the sequence 6,$6 ; 4,4 ; 2,6 ; 3,5$ for both $\mathrm{Au}$ and $\mathrm{Ag}$.

Fig. $5(c, d)$ shows patterns for $A u$ and $A g$, respectively, taken in the vicinity of the 220 f.c.c. reflections, with grain boundary reflections $11,1,0$ and $11, \overline{1}, 0$ on the Ewald sphere. Again, the relative intensities of the grain boundary reflections in both patterns are quite similar. For example, it is seen that the reflections 10,$0 ; 10,4 ; 11,1$ are in a decreasing order of intensity for both $A u$ and $\mathrm{Ag}$.

Fig. 5(e,f) shows patterns for $\mathrm{Au}$ and $\mathrm{Ag}$, respectively, taken in the vicinity of the 400 f.c.c. reflections, with grain boundary reflection $10,10,0$ on the Ewald sphere. Again it is seen that the relative intensities of the grain boundary reflections are approximately the same in both the $\mathrm{Au}$ and $\mathrm{Ag}$ patterns.

As part of this study, the effect of small changes in the boundary structure on the calculated structure factors was examined. It was found that two structures 


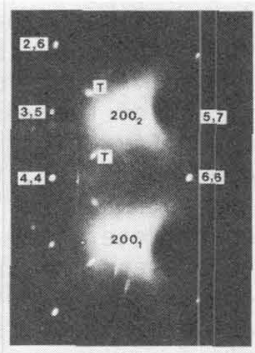

(a)

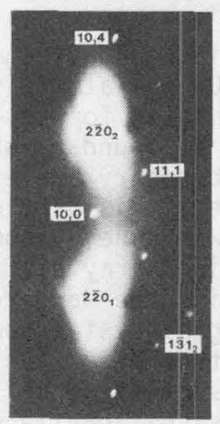

(c)

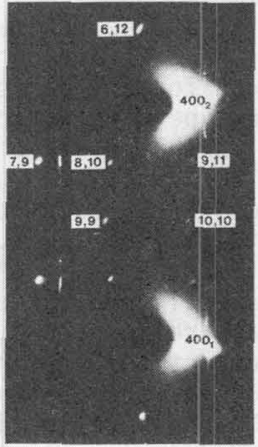

(e)

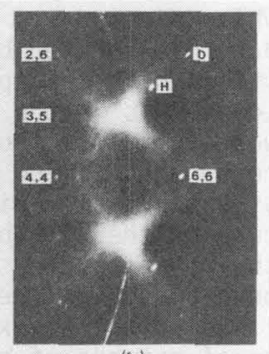

(b)

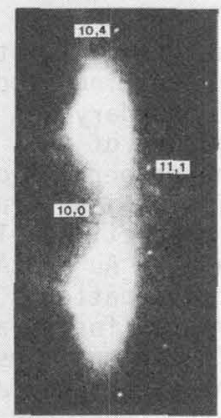

(d)

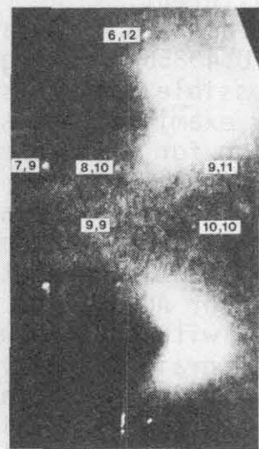

(f)

Figure 5. X-ray diffraction patterns for the $\Sigma=13$ [001] twist boundary taken with $4 \mathrm{hr}$. exposures using synchrotron radiation.

(a) and (b) The 200 region of reciprocal space with grain boundary reflection $6,6,0$ on the Ewald sphere. Reflections due to (1) twins are indicated by $T,(2)$ double diffraction are indicated by $D$ and (3) wavelength harmonics are indicated by $H$. (a) Au (b) Ag.

(c) and (d) The 220 region of reciprocal space with grain boundary reflections $11,1,0$ and $11, \overline{7}, 0^{-}$on the Ewald sphere. (c) Au (d) Ag.

(e) and (f) The 400 region of reciprocal space with grain boundary reflection $10,10,0$ on the Ewald sphere. (e) Au (f) Ag. 
with atomic positions which differ by only $0.005 \mathrm{~nm}$ can give rise to quite different structure factors. The weak reflections are especially sensitive to small changes in structure. Thus it is believed that if two sets of grain boundary structure factor observations are similar, the corresponding structures are also similar.

What can be said now about the structures of the $\mathrm{Au}$ and $\mathrm{Ag}$ boundaries? The similarity between the relative intensities of the grain boundary reflections for the $A u$ and $A g$ boundaries, including observations for $L \neq 0$ as well as $L=0$ (12), suggests that the Au and $\mathrm{Ag}$ boundary structures are also similar. Since most of the observations were made in the $L=0$ plane, this statement is strongest with respect to the structures of the $\mathrm{Au}$ and $\mathrm{Ag} . \Sigma=13$ [001] twist boundary projected onto the boundary plane.

It remains now to discuss why the $\mathrm{Au}$ and $\mathrm{Ag}$ boundary structures are found experimentally to be similar, when computer modelling predicts that they should be different. In the grain boundary study conducted here, what is not known is the concentration of impurities at the boundary plane. Since the sintering together of the two single crystals to produce the bicrystal was carried out in air, it can be expected that either a sulfide or an oxide is present on the surface of the sitver single crystals. After sintering, it is reasonable to expect that there is a second component dissolved in the $\mathrm{Ag}$ in the vicinity of the boundary. There may also be a small amount of $\mathrm{Ag}$ dissolved in the Au bicrystal. Thus the comparison being made in the study may actually be between a Au boundary containing a small amount of $\mathrm{Ag}$ and a $\mathrm{Ag}$ boundary containing a small amount of impurity such as oxygen or sulfur. It is difficult to believe, however, that the presence of impurities can modify the Ag boundary structure, which is actualiy different from the Au structure, and cause it to be similar. This diffraction study has been recently repeated using Au and Ag bicrystals produced by epitaxial growth and sintering under UHV conditions, and the observations are the same as shown in Fig. 5(19). This result indicates that impurities are not strongly influencing the observations in Fig. 5. At present the origin of the disagreement between the results of the $X$-ray experiment and the computer modelling prediction is not understood. However, the similarity of the Au and Ag observations suggests that the fine details of the interatomic potential are not important in determining the boundary structure.

\section{Measurements of Grain Boundary Structural Width(7)}

An important structural characteristic of a grain boundary is its thickness. When discussing thickness, it is necessary to specify what is meant by this term. For example, in the diffusion literature, the term "thickness" refers to the "diffusion width" which is a measure of the width of the boundary region where the diffusing species is highly mobile. Another possible use of the term refers to the "structural width" which is a measure of the depth of penetration of the grain boundary displacement field into the neighboring single crystals. It is expected from theoretical considerations that the magnitude of the structural width will be related to the magnitude of the periodicity in the plane of the boundary (see, for example, ref. 17).

The grain boundary may be represented by a slab of material containing a periodic displacement field which has displaced the atoms from their perfect crystal positions in a certain number of planes on both sides of the boundary plane. Because the number of perturbed planes is sma11, the reflections associated with this region are elongated to give relrods along the direction norma 7 to the boundary plane. If, for example, the displacement field affected only one plane, the grain boundary would give rise to a set of infinitely long relrods which are arrayed along the direction of the periodicity. The actual length of the relrod, therefore, provides a measure of the width of the region containing the displacement field. By combining the results of X-ray diffraction (16) and computer modelling studies (10), it was suggested that the diffraction technique measures the width of the region of large strain at the boundary.

Hagege, Carter, Cosandey and Sass (7) used an electron diffraction technique to measure the length of the relrods from edge-on [001] tilt grain boundaries contained in Au bicrystals. A typical observation is presented in Fig. 6 where the edge-on boundary and its associated streaks are shown. Fig. 7 summarizes al1 the experimental measurements made on the structural width of (100) and (110) 
boundaries. Also shown is the variation of dislocation spacing, $d_{D}$, with $\theta$ given by the Frank formula $d_{D}=|\vec{b}| / 2 \sin \theta / 2$, where $\vec{b}$ is the Burgers vector. (For the (100) boundary plane, $|\vec{b}|=a_{0}$; for the (110) boundary plane, $|\vec{b}|=a_{0} \sqrt{2} / 2$ ).

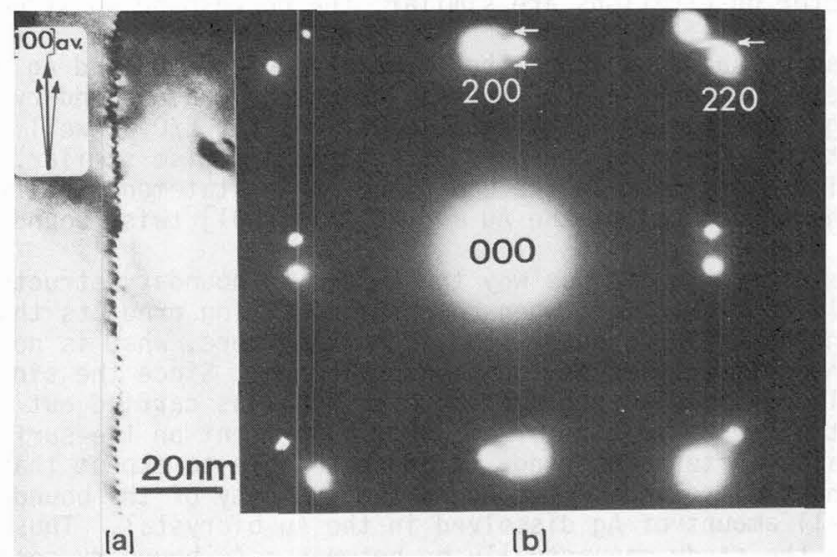

Figure $0 . \quad \theta=10^{\circ}[001]$ (010) grain boundary. (a) Bright-field image showing a periodic array of dislocations along the [100] av. direction. The dislocation spacing is $2 \cdot 3 \pm 0 \cdot 1 \mathrm{~nm}$. (b) Diffraction pattern for (a). The white arrows point to elongated grain boundary reflections. Note the very clear streak in the vicinity of the 220 region.

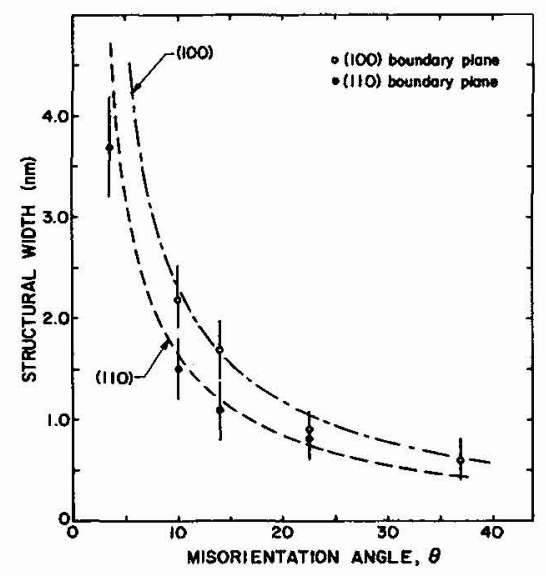

Figure 7. The experimental measurements of grain boundary width plotted versus the misorientation angle. Open and filled circles correspond to the (100) and (110) boundary planes, respectively. These measurements are compared with curves showing the variation of the periodicity in the boundary plane calculated using the Frank formula.

The structural width measurements and the values of the dislocation spacing correlate well, which is an experimental confirmation of the theoretical prediction that the width of the strained region created by a periodic array of dislocations with spacing $d_{d}$, is related to $d_{0}$. The present results suggest that for the cases discussed here the structural width, as determined by electron diffraction, is equal to $d_{D}$. For large angle boundaries it is more appropriate to think of $d_{D}$ as 
being the 0-lattice periodicity, instead of the dislocation spacing.

It is possible that the presence of segregation to the grain boundary will affect the relrod profiles. In order to use the diffraction technique to study the distribution of solute in the vicinity of the boundary plane, it will be important to understand in a quantitative manner the factors that affect the intensity distribution along the relrod.

V. Genera1 Conclusions

A. Diffraction techniques can be used to obtain information on the detailed atomic structure of large angle grain boundaries. In particular it is possible to determine 1) the symmetry and, from this, the translation state of the boundary structure, 2) the projected atomic structure and 3) the structural width of the boundary.

B. The $\Sigma=5\left(\theta=36.9^{\circ}\right)$ and $\Sigma=13\left(\theta=22.6^{\circ}\right)$ [001] twist boundaries in Au were shown to be in the untranslated (coincidence) configuration, by a determination of the boundary symmetry.

C. The projected structure of the $\Sigma=5$ boundary consists of groups of atoms which have undergone large rotations about 0 -elements in the planes immediately adjacent to the boundary. The structure is made up of discontiguous patches of median f.c.c. structure. A similar atomic configuration is present in the $\Sigma=13$ boundary. It is suggested that large rotations about 0 -elements to produce median f.c.c. regions occur in al1 large angle [001] twist boundaries.

D. The atomic structures of the large angle $\Sigma=13$ [001] twist boundary in $\mathrm{Au}$ and $\mathrm{Ag}$ are quite similar. This conclusion is in disagreement with computer modeliing predictions that the structures are quite different. Comparison of the $\Sigma=5$ structure determined by diffraction with that generated by computer modelling al so shows large disagreements.

E. The value obtained for the structural width of [001] tilt boundaries in $\mathrm{Au}$ was shown to be equal in magnitude to the dislocation spacing (0-lattice periodicity) associated with the misorientation of the boundary.

Acknowledgements

This research was supported by the National science Foundation under grant DMR-79-16331 and $i$ ts predecessors. The $X$-ray experiments were carried out at the Corne11 High Energy Synchrotron Source. The use of the central facilities of the Materials Science Center is gratefully acknowledged.

\section{References}

1. T. Schober and R.W. Balluffi, Phil. Mag. 21, 109 (1970).

2. R.C. Pond, D.A. Smith and W.A.T. Clark, J. Micro. 102, 309 (1974).

3. 0.L. Krivanek, S. Isoda and K. Kobayashi, Phil. Mag. 36, 931 (1977).

4. A. Bourret and J. Desseaux, Phil. Mag. A39, 405, 419 (7979).

5. D.Y. Guan and S.L. Sass, Phi1. Mag. 27,1211 (1973).

6. S.L. Sass, T.Y. Tan and R.W. Balluffi, Phil. Mag. 3i, 559 (1975).

7. S. Hagege, C.B. Carter, F. Cosandey and S.L. Sass, Phil. Mag. A45, 723 (1982).

8. R.C. Pond and V. Vitek, Proc. Roy. Soc. Lond. A357, 453 (1977).

9. M. Hashimoto, H. Ichinose, Y. Ishida, R. Yamamoto and M. Doyama, Jap. J. App 1. Phys. 19, 1045 (1980).

10. P.D. Bristowe and S.L. Sass, Acta Meta11. 28, 575 (1980).

11. J. Budai, P.D. Bristowe and S.L. Sass, submitted for publication.

12. J. Budai, A.M. Donald and S.L. Sass, Scripta Meta11. 16, 393 (1982).

13. W. Gaudig and S.L. Sass, Phil. Mag. A39, 725 (1979).

14. P.D. Bristowe and A.G. Crocker, Phi1. Mag. A38, 487 (1978).

15. W. Bollmann, Crystal Defects and Crystalline Interfaces (1970)

Springer-Verlag, New York.

16. J. Budai, W. Gaudig and S.L. Sass, Phi1. Mag A40, 757 (1979).

17. A.H. Cottre11, Dislocations and Plastic Flow in Crystals (1953) Oxford University Press.

18. L.H. Schwartz and J.B. Cohen (1977) Diffraction From Materials, Academic Press.

19. K. Milkove, private communication. 